\title{
Estudio arqueoastronómico en el sitio arqueológico de Yarumela
}

Josué Erubel Ramos Castro ${ }^{1}$

\section{RESUMEN}

El presente proyecto surge como una iniciativa para seguir avanzando en las investigaciones de tipo arqueoastronómíco y de esta forma avanzar en conocimiento de nuestras culturas antepasadas utilizando la información arqueológica hondureña, se desarrolló un estudio de campo, del sitio arqueológico de Yarumela, se realizó, una revisión bibliográfica de otros investigadores, se muestran fotos e imágenes del sitio arqueológico y de sus estructuras. Se realizó un plano topográfico y un mapa georreferenciado para determinar la orientación entre las estructuras, con la cual obtuvimos tablas comparativas de las coordenadas de algunas estructuras de Yarumela, tablas del acimut entre las estructuras y se hicieron cálculos de geometría para determinar el acimut de la salida del sol por el horizonte en los diferentes eventos solares midiendo la altura de las montañas y así determinar si hay algún alineamiento arqueoastronómico entre las estructuras y la salida del sol en los eventos solares, equinoccios y solsticios. Para este estudio sé uso tecnología para planos topográficos y sistemas de información geográfica para análisis de datos de puntos tomados con estación total y GPS, también se utilizó la trigonometría para hacer los cálculos y compararlos con los datos tomados con los de la estación total, esperando obtener el resultado de alineamientos astronómicos y avanzar más en la investigación arqueoastronómica en el país.

Palabras clave: sitios arqueológicos, arqueoastronomía, alineamientos, orientación, acimut, equinoccios, solsticios.

${ }^{1}$ Profesor del Departamento de Arqueo astronomía y Astronomía Cultural, Facultad de Ciencias Espaciales. UNAH: jeramos@unah.edu.hn 


\section{ASBTRACT}

The present project emerges as an initiative to continue advancing in archaeological astronomical research and in this way to advance knowledge of our ancestor cultures using Honduran archaeological information, a field study of the archaeological site of Yarumela was carried out. Bibliographical review of other researchers, photos and images of the archaeological site and its structures are shown. A topographic plane and a georeferenced map were realized to determine the orientation between the structures, with which we obtained comparative tables of the coordinates of some structures of Yarumela, tables of the azimuth between the structures and calculations of geometry were made to determine the azimuth of the Sunrise over the horizon in the different solar events measuring the height of the mountains and thus determine if there is some archeoastronomic alignment between the structures and the sunrise in the solar events, equinoxes and solstices. For this study I use technology for topographic maps and geographic information systems for analysis of points data with total station and GPS, also used the trigonometry to make the calculations and compare them with the data taken with those of the total station, waiting To obtain the result of astronomical alignments and to advance more in the archeoastronomic investigation in the country.

Keywords: Archaeological sites, archeoastronomy, alignments, orientation, azimuth, equinoxes, solstices. 


\section{INTRODUCCIÓN}

En los últimos tiempos la investigación científica en el área de la arqueoastronomía se ha venido desarrollando en nuestro país principalmente por investigadores del departamento de arqueoastronomía y Astronomía Cultural de la Facultad de Ciencias Espaciales, ya que en Honduras existe muchos sitios arqueológicos y que producto de esas investigaciones arqueológicas se ha despertado el interés de hacer investigación pero ya incursionando en la Arqueoastronomía, precisamente en estos sitios que son el pasado cultural y porque no decir nuestros ancestros en ancestros, que desde tiempos a.C regalaron sus conocimientos que hoy poco a poco empezamos a descubrir en varios campos, sobre Arquitectura, Matemática y Astronomía. Por esta razón se quiso investigar uno de los sitios que llama mucho la atención como es el sitio arqueológico de Yarumela, que tiene una existencia casi de 1500 años a.C período formativo (Mandeville J. L., 1997) y que ha sido considerado como uno de los lugares más antiguos del corredor mesoamericano y que precisamente se encuentra localizado entre los departamentos de Comayagua y La Paz. Después de varias visitas al lugar con alumnos de las asignaturas de Introducción a la Arqueoastronomía AQA-111, Arqueoastronomía Maya AQA-112 y alumnos del Diplomado de Arqueoastronomía Maya despertó la curiosidad de ir más a fondo en la investigación en este lugar tan antiquísimo, según los planos encontrados en la bibliografía de otros autores que investigaron el lugar desde un punto de vista arqueológico y la forma en que se encuentran ubicadas las estructuras daban la impresión de estar alineadas entre ellas y con la salida del sol en los eventos solares. Se cree que si investigando quienes ocuparon el sitio en su momento más asombroso y que realmente tenían un conocimiento avanzado era posible que sus construcciones estuvieran alineadas.

\section{METODOLOGÍA}

El enfoque de esta investigación es cuantitativo porque busca resolver un problema basado en el análisis de la orientación de cuatro estructuras, que a simple vista parecen estar orientadas y alineadas con la salida del sol, para lo cual se midieron dos variables acimut de la estructura, acimut de la salida del sol por horizonte y altura de las montañas en el horizonte punto cardinal este, por donde el sol sale en los diferentes eventos solares equinoccios y solsticios. Estas mediciones se realizaron con aparatos y herramientas especiales como estación total para medir altura del horizonte y acimut entre estructuras y así determinar si esta estructura tiene una relación 
arqueoastronómica en el análisis con un plano topográfico que se elaboró con las mediciones obtenidas. El proceso se inició con la exploración de fuentes bibliográficas del sitio arqueológico de Yarumela. Las visitas de campo al sitio fueron con el propósito de reconocer el área de estudio, toma de fotografías y toma de coordenadas con Sistema de Geo Posicionamiento Global (GPS) de las estructuras en estudio y otras, con el objetivo de elaborar un mapa georreferenciado de las estructuras más importantes para el estudio y análisis de sus orientaciones, se procedió hacer cálculos de trigonometría para calcular el acimut de las estructuras utilizando la altura de la montaña que se midió con la estación total. Con toda esta información se hizo un análisis de las estructuras tanto en el mapa georreferenciado y el plano topográfico para determinar la orientación de las estructuras y la posible alineación con la salida del sol en los días de los equinoccios y solsticios.

\section{RESULTADOS}

\section{Sitio arqueológico Yarumela}

Durante el período formativo (1000 a.C. a 250 d.C) en el centro de Honduras en el sitio arqueológico de Yarumela, se desarrolló una sociedad indígena que formó la base de la cultura Lenca encontrada por los conquistadores en 1537 (Dixon, 1991). Los hallazgos encontrados en el sitio arqueológico de Yarumela el cual corresponde al periodo clásico formativo mesoamericano y que fue pronto fue reconocido por ciertos investigadores porque en este lugar hubo un contacto temprano entre Mesoamérica y Perú, donde florecieron las culturas como la olmeca y la chavin respetivamente hace más de 1,500 años a.C. Este sitio es conocido localmente como chilcal, y en vista de que hubo una relación con estas culturas que ya para el entonces tenían un conocimiento avanzado en la observación del cielo a simple vista. Se cree que Yarumela era el asentamiento del cacicazgo dominante de todo el valle durante los tiempos del formativo tardío 400 a.C. Hasta por lo menos 250 años d.C. (Dixon 1989) citado en (Mandeville J. , 1997) Finalmente la arquitectura monumental en tal escala nunca fue practicada después del siguiente período clásico y, eventualmente fue abandonada por completo afínales del período postclásico, Dixon 1989 ( Mandeville J. , 1997). El sitio del Formativo tardío en Yarumela está dominado por la estructura 101, de $20 \mathrm{~ms}$ de altura que forma una "C" cara hacia el este y con estructuras de 6 ms de alto y la 103 y la 104 al sur y norte, respectivamente. Dentro de esta área, se enmarca una plaza central de $9 \mathrm{~ms}$ de altura la estructura 102 en las riveras del Humuya y 4 montículos de $3 \mathrm{~ms}$ de altura. Además, en agrupaciones menos 
obvias y esparcidas por todo el sitio, hay 10 montículos y varias plataformas destruidas ( Mandeville J. , 1997).

Ubicación geográfica de Yarumela El emplazamiento arqueológico Yarumela está ubicado en el valle de Comayagua que se extiende por $1.5 \mathrm{~km}$ y una altitud de $600 \mathrm{~ms}$ sobre el nivel del mar. Está asentado en la ribera occidental del Río Humuya. El clima en este paraje comprende una estación seca de enero a abril y una temporada lluviosa de mayo a diciembre. Sin embargo, Yarumela es algo más caliente y seco debido al efecto de sombre fluvial que provoca la cercanía de las montañas que tienen una altitud de más de 1000 ms. (Dixon, 1991). Las coordenadas geográficas del sitio arqueológico de Yarumela son: Latitud $14.3637^{\circ} \mathrm{N}$ y Longitud $87.65^{\circ} \mathrm{W}$.

Se desarrolló un mapa georreferenciado del sitio arqueológico de Yarumela con los puntos de las estructuras más importantes para este estudio, estos puntos fueron tomados con GPS, en el mismo se puede observar que las estructuras 101 y 102 están orientadas de este a oeste y las 103,102 y 104 de sur a norte. Hay otros montículos o estructuras que fueron tomadas en consideración como la 106 y 108 pero la 111, 107 con más referencia para los solsticios de invierno y verano respectivamente. Ver Tabla 1 y Fig. 1

Tabla 1. Coordenadas Geográficas de las estructuras.

\begin{tabular}{|c|c|c|c|}
\hline Estructuras & Latitud & Longitud & $\begin{array}{c}\text { Altura de la } \\
\text { estructura }\end{array}$ \\
\hline 101 & $14^{\circ} 21^{\prime} 49.20304^{\prime \prime}$ & $87^{\circ} 39^{\prime} 2.999^{\prime \prime}$ & $20 \mathrm{~ms}$. \\
\hline 102 & $14^{\circ} 21^{\prime} 49.3560^{\prime \prime}$ & $87^{\circ} 38^{\prime} 51.26568^{\prime \prime}$ & $9 \mathrm{~ms}$ \\
\hline 103 & $14^{\circ} 21^{\prime} 35.07976^{\prime \prime}$ & $87^{\circ} 38^{\prime} 48.84185^{\prime \prime}$ & $6 \mathrm{~ms}$ \\
\hline 104 & $14^{\circ} 22^{\prime} 3.95220^{\prime \prime}$ & $\begin{array}{c}87^{\circ} 38^{\prime} \\
50.456642^{\prime \prime}\end{array}$ & $6 \mathrm{~ms}$ \\
\hline 106 & $14^{\circ} 21^{\prime} 48.2822^{\prime \prime}$ & $87^{\circ} 38^{\prime} 57.44038^{\prime \prime}$ & $4 \mathrm{~ms}$. \\
\hline 108 & $14^{\circ} 21^{\prime} 50.05940^{\prime \prime}$ & $87^{\circ} 38^{\prime} 55.8635^{\prime \prime}$ & $3 \mathrm{~ms}$ \\
\hline 111 & $14^{\circ} 21^{\prime} 45.88626^{\prime \prime}$ & $87^{\circ} 38^{\prime} 53.9417^{\prime \prime}$ & No se conoce su altura \\
\hline 107 & \multicolumn{3}{|c|}{ No se encontró la estructura } \\
\hline $\begin{array}{l}\text { Coordenadas Geograficas y altura de los monticulos del Stio Arqueologicode Yarumela Tomados por el } \\
\text { autor con GPS. Fuente elboracion propia }\end{array}$
\end{tabular}


Figura 1. Mapa del Sitio Arqueológico de Yarumela con algunas de las estructuras georreferenciadas importantes para el estudio. Fuente elaboración propia

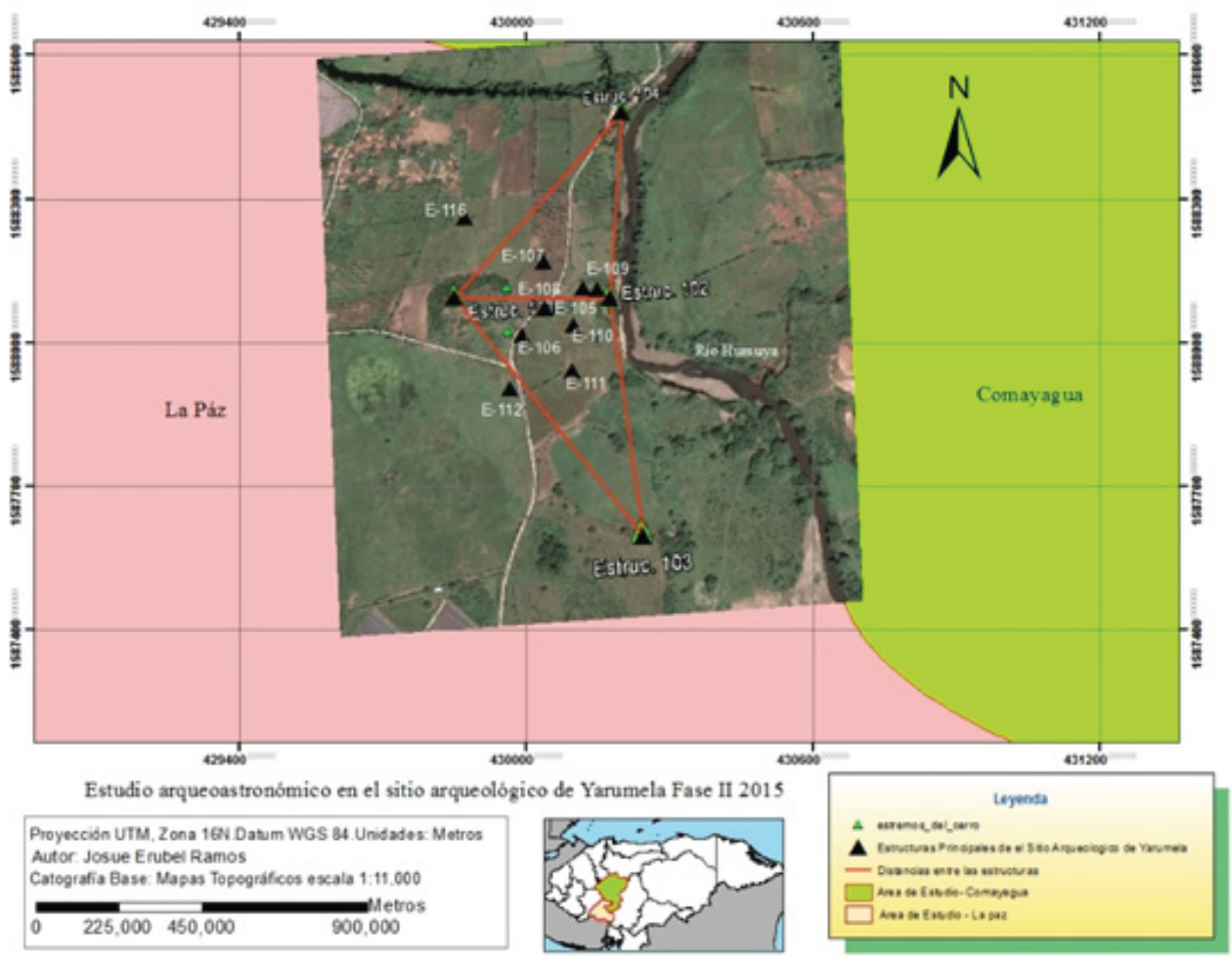

La arquitectura del sitio sobre el nivel del suelo cubre aproximadamente 30 has. Sobre una terraza del pleistoceno de $10 \mathrm{~ms}$. de altura sobre el curso actual del Río Humuya ( Mandeville J. , 1997). Según Ephrain G. Squier, que visitó el sitio de Yarumela mientras inspeccionaba el valle de Comayagua describió el sitio con un estado de Conservación casi intacto que contrasta con el deterioro actual. ( Mandeville J. , 1997) Según Squier la Arquitectura era de forma rectangular y colocada con mucha delicadeza en referencia con los puntos cardinales. Los datos etnográficos utilizados para establecer la conducta reflejada en la cultura material del periodo formativo (Mandeville J. , 1986). Ver Tabla 2.

Se tomó fotos en el momento en el que el sol se levanta por el horizonte para determinar la Arqueoastronomía y el significado de la posición horizontal de la salida en relación a un particular alineamiento de los montículos durante los solsticios y equinoccios Ver Fig. 2,3 4, y 5. 
Tabla 2. Características de cada estructura

\begin{tabular}{|c|c|l|}
\hline $\mathbf{N}^{\circ}$ & Estructura & \multicolumn{1}{c|}{ Características } \\
\hline 1 & 101 & $\begin{array}{l}\text { Debajo de esta estructura se conserva un registro muy } \\
\text { claro de una estructura residencial o ceremonial de la } \\
\text { elite, siendo la evidencia más antigua de la periferia del } \\
\text { sudeste mesoamericano. Esta superestructura medía } \\
\text { aproximadamente 32ms. de norte a sur por 20ms de } \\
\text { este a oeste. En su eje largo con cara a la plaza } \\
\text { principal hacía el este y una altura de 20ms. }\end{array}$ \\
\hline 2 & 102 & $\begin{array}{l}\text { Durante el formativo tardío se intensificó la } \\
\text { construcción alrededor de la Plaza principal definiendo } \\
\text { más este espacio público y colocando a esta estructura } \\
\text { en su lado este. Un núcleo de tierra de 4ms. de altura } \\
\text { del periodo formativo medio fue agregado unas cuatro } \\
\text { veces durante 600 años elevando la cima del } \\
\text { montículo hasta 9 ms. sobre la plaza alrededor del año } \\
250 \text { a.C }\end{array}$ \\
\hline 3 & 103 & $\begin{array}{l}\text { Estas dos estructuras 103, 104 y la 102 también } \\
\text { muestran la aparición de la arquitectura monumental } \\
\text { en Yarumela, se encuentran alineadas a lo largo del río } \\
\text { Humuya, descubrieron núcleos sustanciales de arcilla } \\
\text { y cieno del sedimento de aluvión de dos o cuatro } \\
\text { metros de alto, rematados con pisos con repello } \\
\text { preparado con cal. }\end{array}$ \\
\hline 4 & 104 & $\begin{array}{l}\text { Parece haber sido construida durante el período } \\
\text { formativo medio y se mantuvo en pie sin cambios } \\
\text { durante los siguientes 600 años o más hasta el } \\
\text { abandono del sitio. Fue construido con una altura de 4 } \\
\text { ms. }\end{array}$ \\
\hline 5 & 108 & $\begin{array}{l}\text { De estas tres estructuras no se encontró ningúna } \\
\text { información en la bibliografía consultada más que } \\
\text { en los planos del sitio }\end{array}$ \\
\hline 8 & 107 & 106 \\
\hline Mandeville J. , 1997)
\end{tabular}

Esta tabla muestra información de las características de cada estructura encontrada por los arqueólogos que hicieron la investigación (Mandeville J. L., 1997). 
Figura 2. Salida del sol en el equinoccio de primavera 20 de marzo 2014

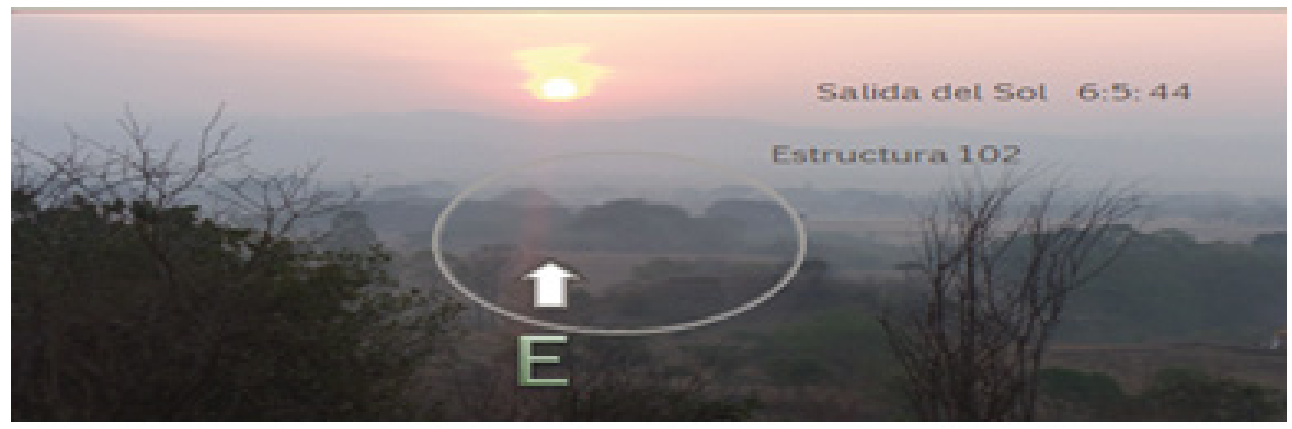

Estructura 102 vista desde la estructura 101, foto tomadas por el autor.

Figura 3. Salida del sol en el Solsticio de Verano 21 de junio 2014

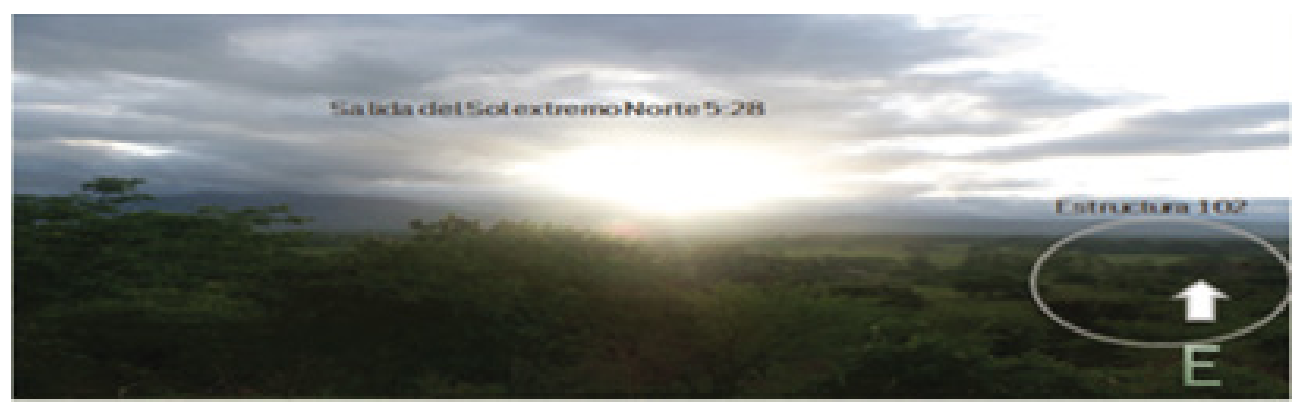

Foto tomada desde la estructura 101 Tomada por el autor.

Figura 4. Salida del sol en el equinoccio de otoño 22 de septiembre 2014

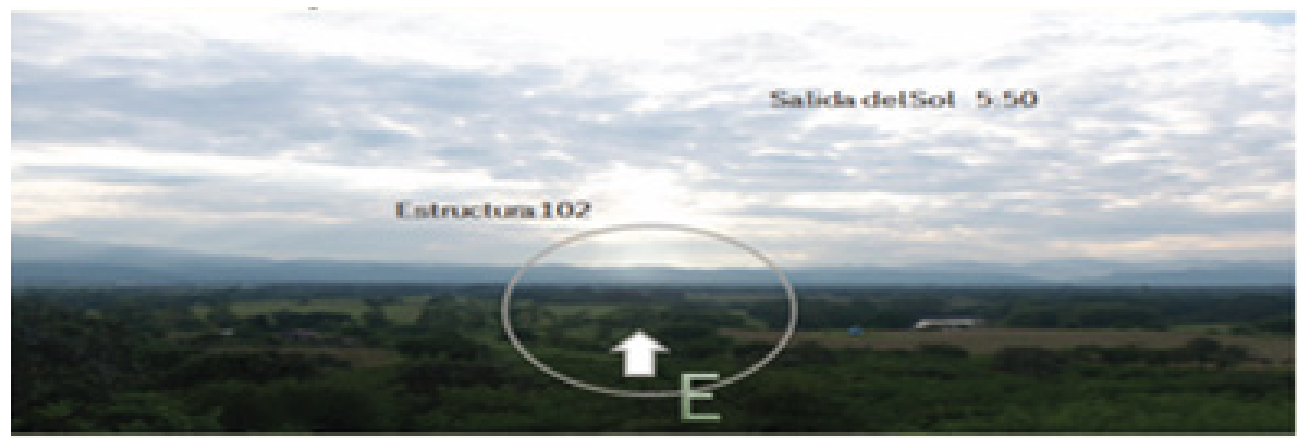

Estructura 102 vista desde la estructura 101. Foto tomada por el autor 
Fotos de las estructuras más sobresalientes del sitio arqueológico de Yarumela

Figura 5. Fotos tomadas de los montículos en la investigación del Sitio Arqueológico de Yarumela en su estado actual. Fotos(a) Estructura 101, Foto (b) Estructura 102, Foto (c) Estructura 103 y foto (d) Estructura (104), Tomadas por el autor.

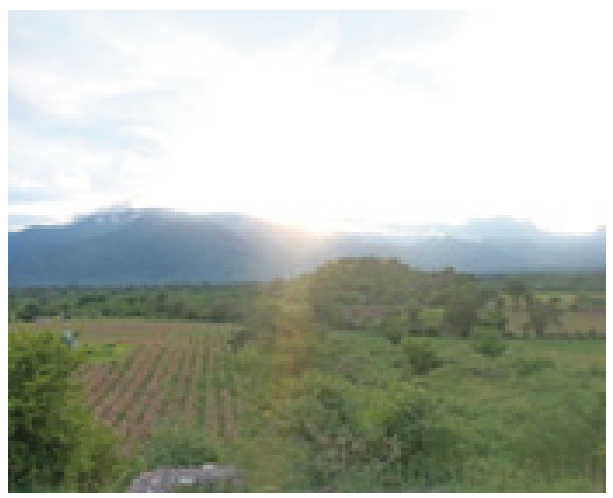

Foto (a)

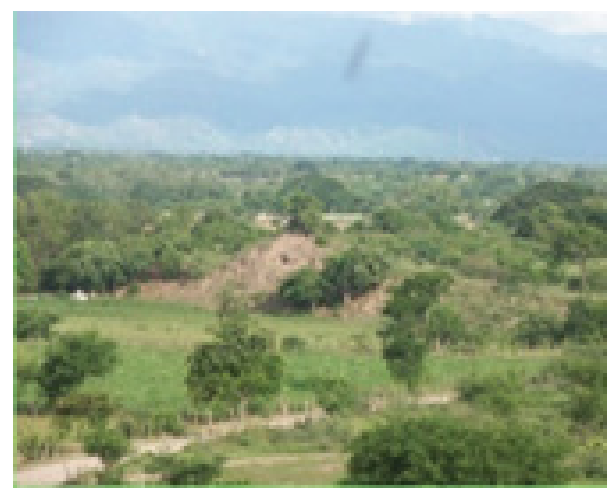

Foto (c)

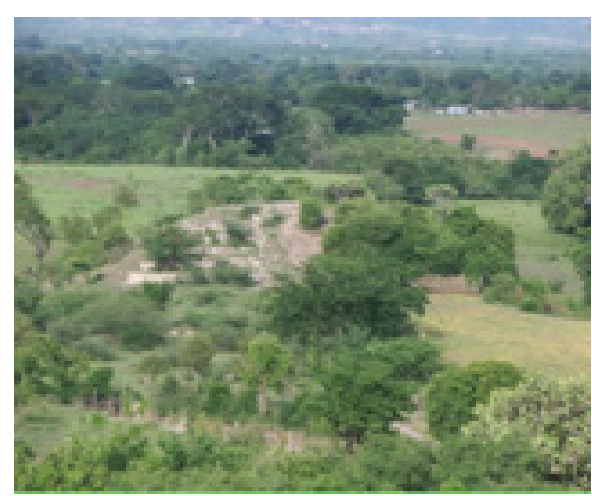

Foto (b)

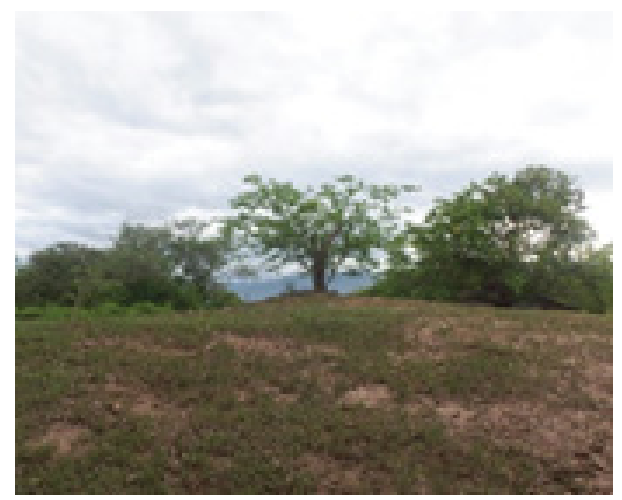

Foto (d)

De las estructuras 111 y 107 no se logró tomar fotos porque actualmente han sido dañadas por los cultivos sembrados en el lugar, sin embargo, se hizo un mapa topográfico para medir el acimut de cada una de las estructuras 101,102, 103, 104, 111, 106, 108 y 110, no así de la estructura 107 que no fue posible ubicar por se presume que fue destruida por los cultivadores del lugar para sus siembras. Ver Tabla 3 
Tabla 3. Acimut entre estructuras

\begin{tabular}{|l|c|c|c|c|c|}
\hline \multirow{2}{*}{ Lugar } & \multicolumn{3}{|c|}{ Sitio arqueológico de Yarumela Levantamiento, Topográfico con } \\
estación total
\end{tabular}

Tabla 4. Altura de la montaña Fuente elaboración propia

\begin{tabular}{|c|c|c|c|c|}
\hline Lugar & \multicolumn{3}{|c|}{$\begin{array}{c}\text { Sitio arqueológico de Yarumela } \\
\text { (alturas de la montaña en el este) }\end{array}$} \\
\hline $\begin{array}{c}\text { Evento } \\
\text { solar }\end{array}$ & $\begin{array}{c}\text { Equinoccio } \\
\text { de primavera }\end{array}$ & $\begin{array}{c}\text { Solsticio de } \\
\text { verano }\end{array}$ & $\begin{array}{c}\text { Equinoccio } \\
\text { de otoño }\end{array}$ & $\begin{array}{c}\text { Solsticio de } \\
\text { invierno }\end{array}$ \\
\hline Altura & $2^{\circ} 07^{\prime} 20^{\prime \prime}$ & $4^{\circ} 02^{\prime} 43^{\prime \prime}$ & $2^{\circ} 07^{\prime} 20^{\prime \prime}$ & $\begin{array}{c}2^{\circ} 38^{\prime} 42 \\
\text { No se pudo } \\
\text { medir }\end{array}$ \\
\hline
\end{tabular}


La tabla anterior muestra la altura del horizonte por donde saldrá el sol en el equinoccio de primavera, otoño, solsticio de verano e invierno. Para conocer el acimut de la salida del sol y demostrar la alineación que existe entre las estructuras 101 y 102 con la salida del sol y la relación de las demás estructuras 103, 104 y ahora 111 y 107 . En el caso de la estructura 107 y 111 se tomó como referencia Plano del Sitio Arqueológico de Yarumela por Joesink Mandeville, y de esta forma medir el acimut de las estructuras y luego la altura de las montañas en el horizonte Este. Ver Fig. 6

Fig. 6. Plano del Sitio Arqueológico de Yarumela por: Joesink Mandeville Con agregados del estudio del autor para el estudio Arqueoastronómíco ( Mandeville J. , 1997)

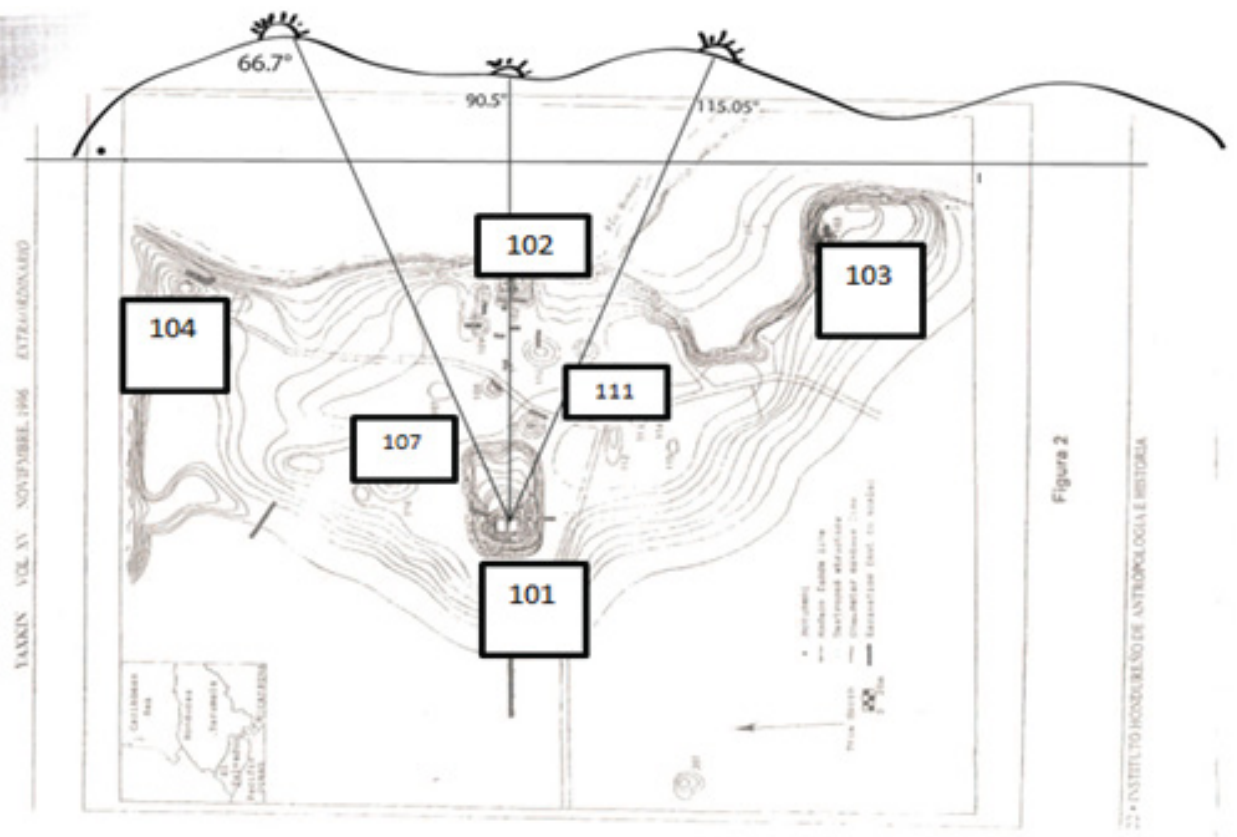

\section{Trigonometría}

El coseno de un lado es igual al producto de los cosenos de los otros dos, más el producto de sus senos multiplicado por el coseno del ángulo comprendido. (Berrecoso, Ramírez , Enríquez Salamanca, \& Pérez Peña, 2003) Para determinar los resultados de la medición con la estación total en cuanto al acimut de las estructuras y la salida del sol en el día del equinoccio de primavera y de otoño, y solsticios de invierno y verano se aplicó y desarrolló la siguiente fórmula. 


\section{Fórmula}

$\cos (90-\delta)=\cos (90-\varphi) \cos (90-h)+\operatorname{sen}(90-\varphi)+\operatorname{sen}(90-\varphi) \operatorname{sen}(90-h) \cos z$

Transformando esta fórmula a trigonometría básica de los senos obtenemos. $\operatorname{sen} \delta=\operatorname{sen} \varphi \operatorname{sen} h+\cos \delta \cos \varphi \cos h \cos z$

Despejando para cos z

$$
\operatorname{Cos} z=\frac{\operatorname{Sen} \delta-\operatorname{Sen} \varphi \operatorname{sen} h}{\operatorname{Cos} \varphi \operatorname{Cos} h}
$$

\section{Cálculos para los equinoccios}

Para determinar el acimut de la salida del sol se tomó la altura de la montaña medida que se obtuvo con la estación total y se aplicó y desarrollo la siguiente fórmula para cada uno de los eventos solares.

\section{Datos}

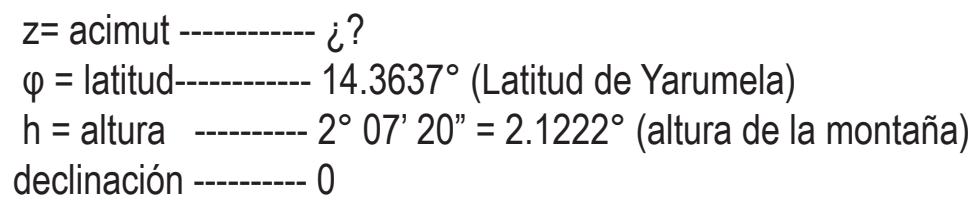

$\operatorname{Cos} z=\frac{\operatorname{Sen} \delta-\operatorname{Sen} \varphi \operatorname{sen} h}{\operatorname{Cos} \varphi \operatorname{Cos} h}$

\section{Sustituyendo}

$\operatorname{Cos} z=$

$$
\operatorname{Sen} 0^{\circ}-\operatorname{Sen}\left(14.3637^{\circ}\right) \operatorname{Sen}\left(2.1222^{\circ}\right)
$$

$\operatorname{Cos}\left(14.3637^{\circ}\right) \operatorname{Cos}\left(2.1222^{\circ}\right)$

$(-0.2480)(0.0370)$

$\operatorname{Cos} z=$

$$
(0.9687)(0.9993)
$$


$(-0.009176)$

$\operatorname{Cos} z=$

$\operatorname{Cos} z=-0.009479$

\section{(0.96802)}

Sacando el coseno inverso

$z=90.5^{\circ}$ (acimut)

El acimut por donde sale el sol en las coordenadas geográficas de Yarumela el día del equinoccio de primavera es de $90.5^{\circ}$ y también en el equinoccio de otoño para el año 2014.

Figura 7. Representación de coordenadas horizontales y la declinación del sol en el punto de salida por el este el día del equinoccio de primavera

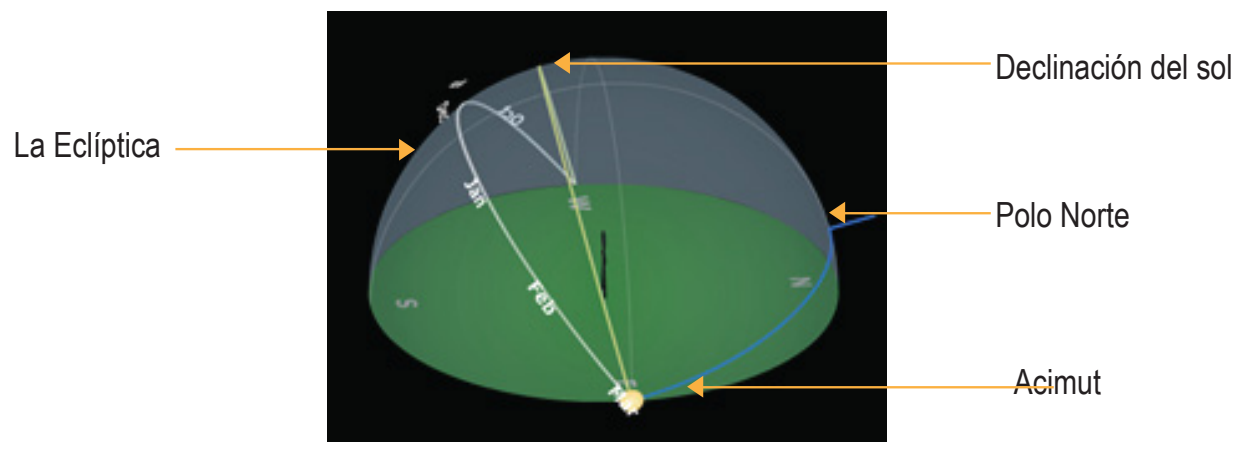

Astronomy Education At the University of Nebraska- Lincolh, 2010

Figura 8. Representación de coordenadas horizontales y la declinación del sol en el punto de salida por el este el día del equinoccio otoño respectivamente

La Eclíptica

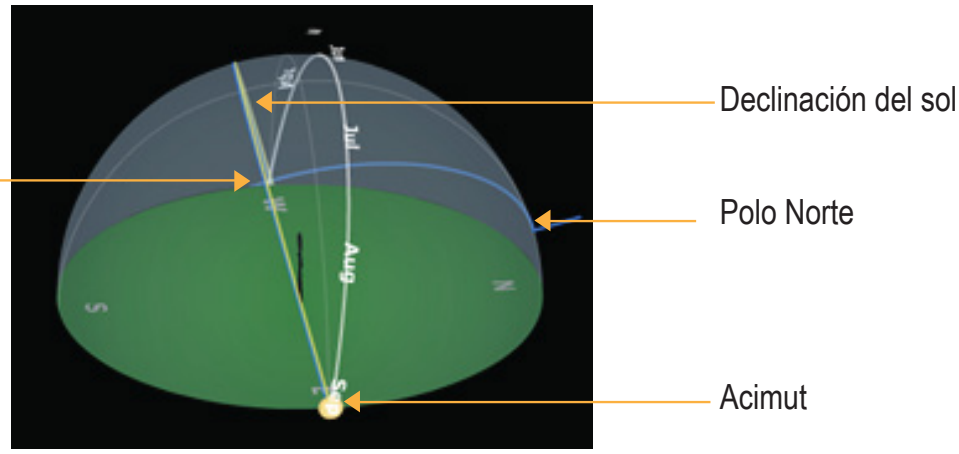

Astronomy Education At the University of Nebraska- Lincolh, 2010 
Cálculos para el solsticio de verano

\section{Datos}

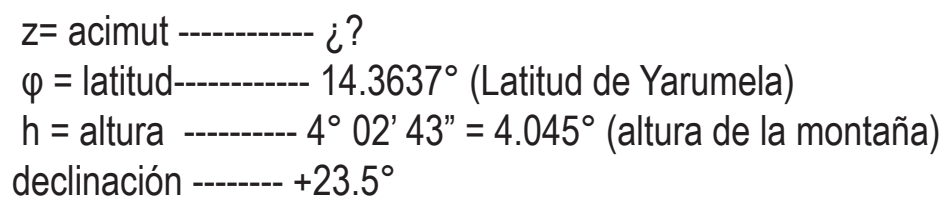

$\operatorname{Sen} \delta-\operatorname{Sen} \varphi \operatorname{sen} h$

$\operatorname{Cos} z=$

$$
\operatorname{Cos} \varphi \operatorname{Cos} h
$$

\section{Sustituyendo}

$\operatorname{Cos} z=\frac{\operatorname{Sen} 23.47-\operatorname{Sen}\left(14.3637^{\circ}\right) \operatorname{Sen}\left(4.045^{\circ}\right)}{\operatorname{Cos}\left(14.3637^{\circ}\right) \operatorname{Cos}\left(4.045^{\circ}\right)}$

$\operatorname{Cos} z=$

$$
0.3983-0.2480(0.0705)
$$

$$
(0.9687)(0.9975)
$$

$(0.380816)$

$\operatorname{Cos} z=$

$(0.966278)$

$\operatorname{Cos} z=0.394106$

\section{Sacando el coseno inverso}

$z=66.7^{\circ}$ (acimut)

El acimut por donde sale el sol en las coordenadas geográficas de Yarumela el día solsticio de verano es de $66.7^{\circ}$ para el año 2014. 
Figura 9. Representación de coordenadas horizontales y la declinación del sol en el punto de salida el día del solsticio verano

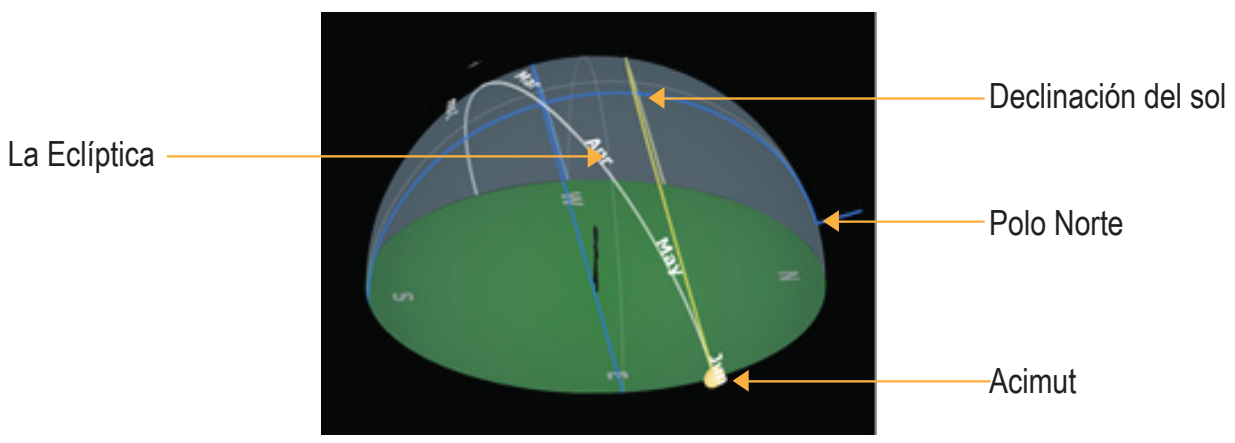

Astronomy Education At the University of Nebraska- Lincolh, 2010

Cálculos para el solsticio de invierno

\section{Datos}

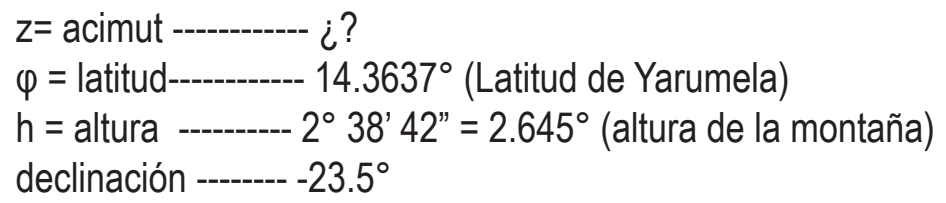

$\operatorname{Sen} \delta-\operatorname{Sen} \varphi \operatorname{sen} \mathrm{h}$

$\operatorname{Cos} z=\frac{\operatorname{Cos} \varphi \cos h}{\operatorname{sen} \delta-\operatorname{Sen} \varphi \operatorname{sen} h}$

\section{Sustituyendo}

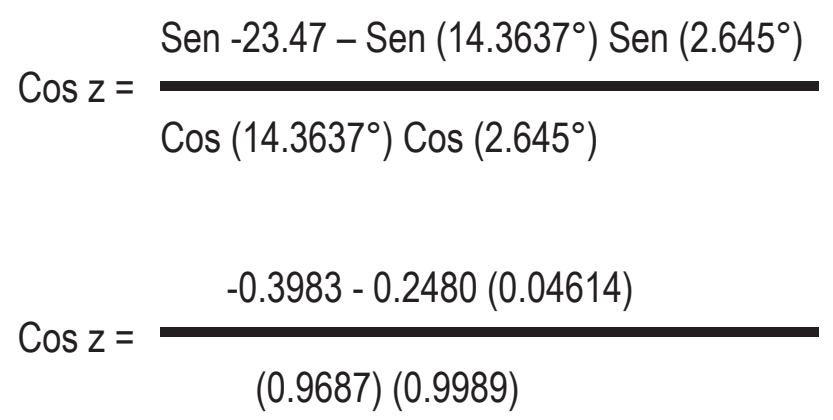


$-(0.40974)$

$\operatorname{Cos} z=$

$\operatorname{Cos} z=-0.42234$

$$
(0.967634)
$$

Sacando el coseno inverso

$z=115.05^{\circ}$ (acimut)

El acimut por donde sale el sol en las coordenadas geográficas de Yarumela el día solsticio de invierno es de $115.05^{\circ}$ para el año 2014.

Al terminar los cálculos de acimut con fórmulas y acimut de estructuras según su posición con respecto al norte magnético, se obtuvieron los resultados de la tabla 5.

Figura 10. Representación de coordenadas horizontales y de la declinación del sol en el punto de salida el día del solsticio invierno

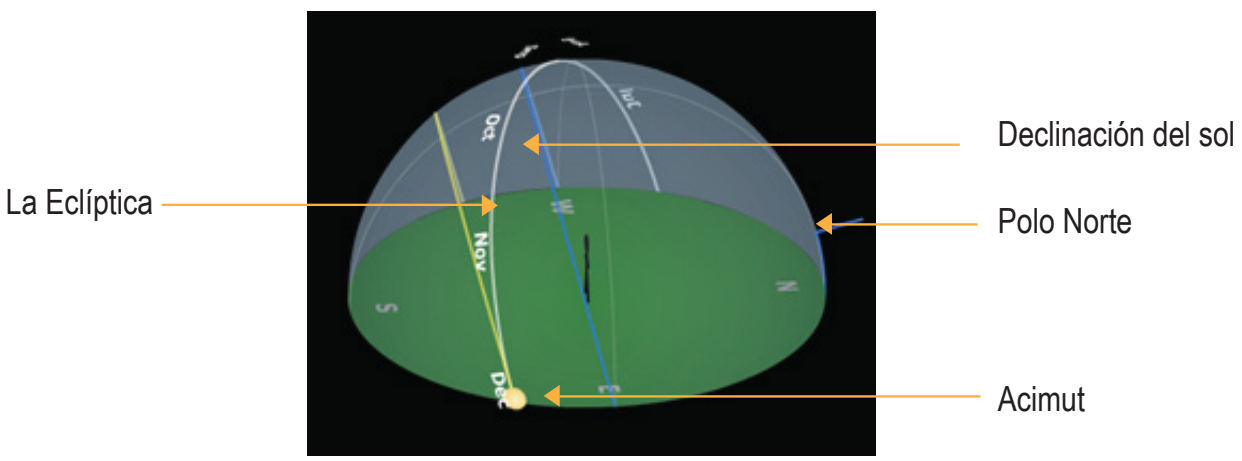

Astronomy Education At the University of Nebraska- Lincolh, 2010

El Stellarium es un software que permite a las personas simular un planetario en su propia computadora, es software libre y está disponible para los principales sistemas. En las siguientes imágenes buscamos proyectar los eventos de equinoccios y solsticios para el año 400 a.C. para observar la similitud con los cálculos en el 2014 Ver Fig. 11, 12, 13, 14,15 y 16. 
Tabla 5. Tabla de comparación entre cálculos del acimut por fórmula y mediciones con estación total

\begin{tabular}{|c|c|c|c|c|}
\hline $\begin{array}{l}\text { Evento } \\
\text { solar }\end{array}$ & $\begin{array}{l}\text { Cálculo de } \\
\text { acimut la } \\
\text { con } \\
\text { fórmula }\end{array}$ & $\begin{array}{c}\text { Acimut } \\
\text { de las } \\
\text { estructuras }\end{array}$ & $\begin{array}{l}\text { Estructuras } \\
\text { relacionadas }\end{array}$ & Observaciones \\
\hline $\begin{array}{c}\text { Equinoccio } \\
\text { de } \\
\text { primavera }\end{array}$ & $90.5^{\circ}$ & $92.3^{\circ}$ & 101 y 102 & \\
\hline $\begin{array}{l}\text { Solsticio } \\
\text { de verano }\end{array}$ & $66.7^{\circ}$ & $66.5^{\circ}$ & 101 y 107 & $\begin{array}{l}\text { Se hizo el } \\
\text { cálculo } \\
\text { aproximado } \\
\text { utilizando el } \\
\text { plano de } \\
\text { Joesink } \\
\text { Mandeville }\end{array}$ \\
\hline $\begin{array}{l}\text { Equinoccio } \\
\text { de otoño }\end{array}$ & $90.5^{\circ}$ & $92.3^{\circ}$ & 101 y 102 & \\
\hline $\begin{array}{c}\text { Solsticio } \\
\text { de invierno }\end{array}$ & $115.05^{\circ}$ & $114.5^{\circ}$ & 101 y 111 & $\begin{array}{l}\text { Se hizo el } \\
\text { cálculo } \\
\text { aproximado } \\
\text { utilizando el } \\
\text { plano de } \\
\text { Joesink } \\
\text { Mandeville }\end{array}$ \\
\hline
\end{tabular}

Esta tabla muestra una comparación entre los cálculos que se hicieron con formula de trigonometría básica de los senos y la medición del acimut tomando como referencia el norte magnético que se realizó utilizando una estación total. Por: el autor

Figura 11. Muestra la salida del sol en los equinoccios para el año 400 a.C a una altura de $2^{\circ} 07^{\prime} 23^{\prime \prime}$ con un acimut de $92^{\circ} 33^{\prime} 14^{\prime \prime}$, se utilizó el programa Stellarium 0.11.3

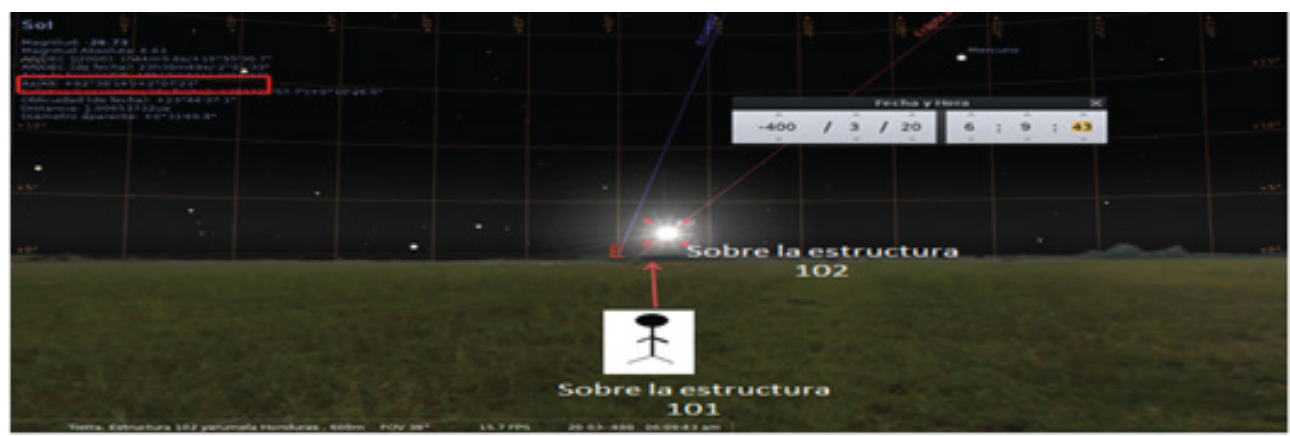


Figura 12. Muestra la salida del sol en los equinoccios para el año 2014 d.C a una altura de $2^{\circ} 07^{\prime} 27^{\prime \prime}$ con un acimut de $92^{\circ} 33^{\prime} 14^{\prime \prime}$, se utilizó el programa Stellarium 0.11 .3

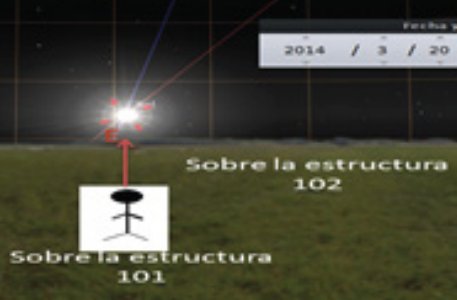

Figura 13. Muestra la salida del sol en el solsticio de verano para el año 400 a.C a una altura de $4^{\circ} 02^{\prime} 43^{\prime \prime}$ con un acimut de $66^{\circ} 41^{\prime} 37^{\prime \prime}$, se utilizó el programa Stellarium 0.11 .3

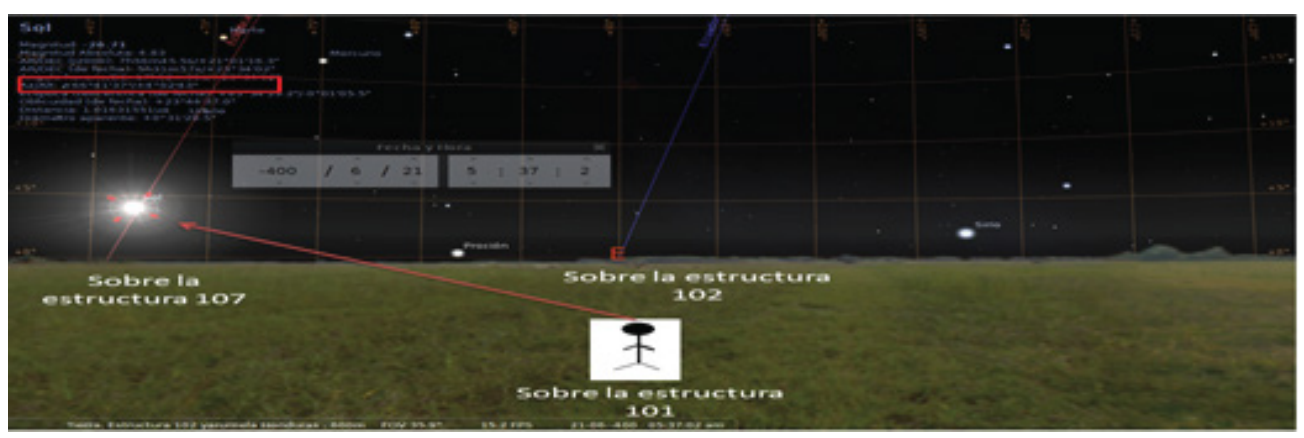

Figura 14. Muestra la salida del sol en el solsticio de verano para el año 2014 d.C a una altura de $4^{\circ} 02^{\prime} 9^{\prime \prime}$ con un acimut de $66^{\circ} 49^{\prime} 41^{\prime \prime}$, se utilizó el programa Stellarium 0.11 .3

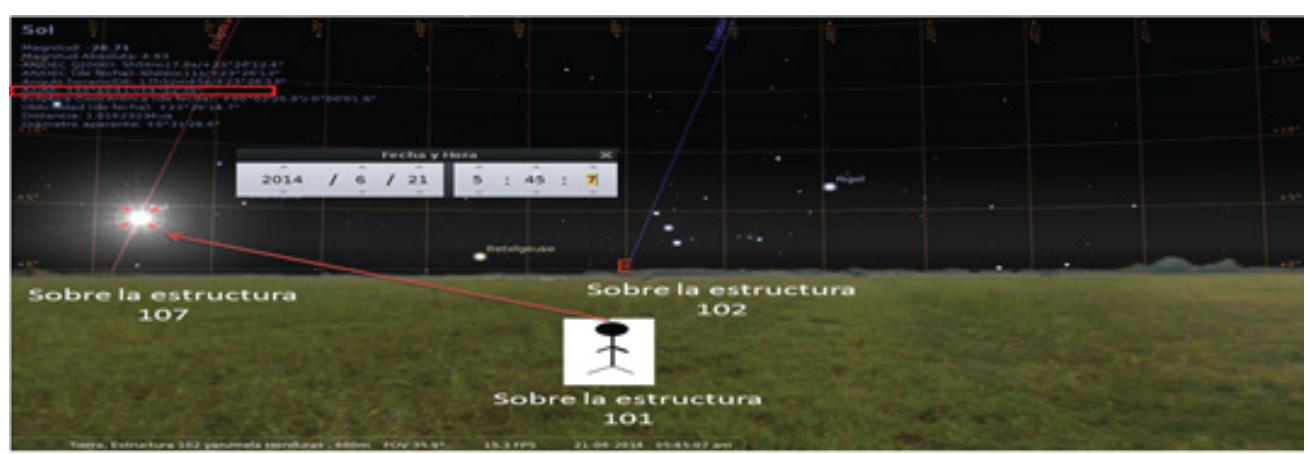


Figura 15. Muestra la salida del sol en el solsticio de invierno para el año 400 a.C a una altura de $2^{\circ} 38^{\prime} 44^{\prime \prime}$ con un acimut de $115^{\circ} 12^{\prime} 60^{\prime \prime}$, se utilizó el programa Stellarium 0.11.3

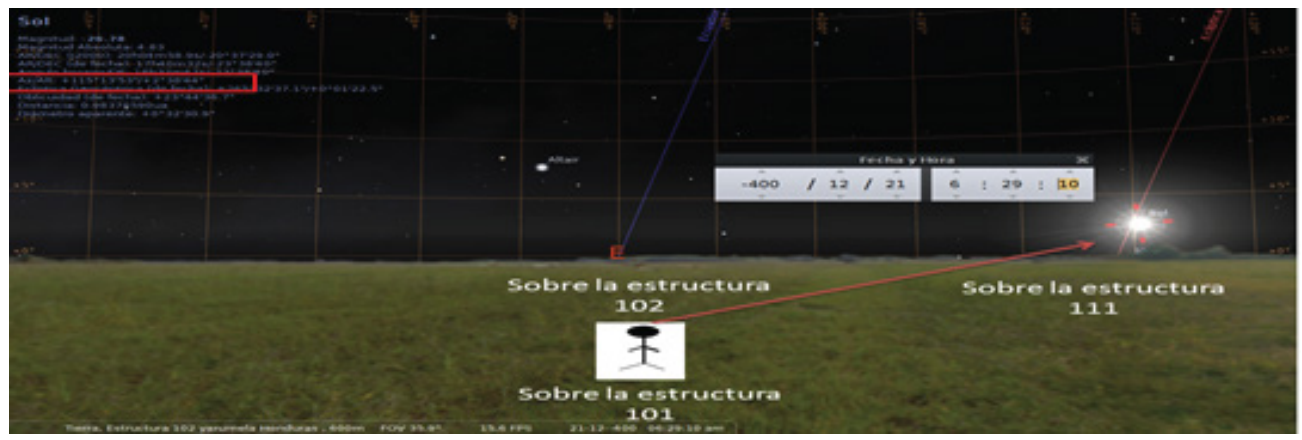

Figura 16. Muestra la salida del sol en el solsticio de invierno para el año 2014 d.C a una altura de $3^{\circ} 18^{\prime} 11^{\prime \prime}$ con un acimut de $115^{\circ} 12^{\prime} 60^{\prime \prime}$, se utilizó el programa Stellarium 0.11.3

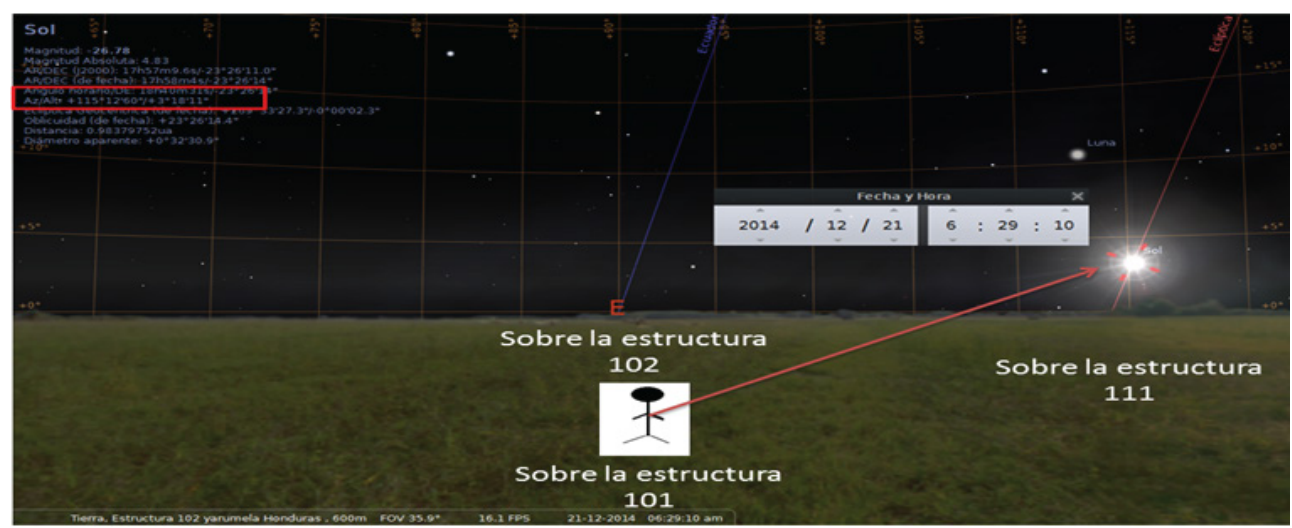

\section{DISCUSIÓN}

En esta investigación se buscó determinar el posible alineamiento de las estructuras que en primera instancia parecían estar alineadas. Es impresionante como se observa la salida del sol por el punto cardinal este en los días del equinoccio posiblemente alineado con las dos estructuraras más importantes por su forma especial de orientación, aunque existen otras estructuras que no están alineadas con los solsticios como se pretendía determinar. 


\section{CONCLUSIONES}

1. Para el primer objetivo podemos determinar que entre las estructuras 101 y 102 hay una orientación de este a oeste, y que el sol se levanta por encima de las montañas a un acimut muy cercano a los $90^{\circ}$, lo cual nos permite decir que si hay un posible alineamiento entre estas dos estructuras y la posición del sol en el momento de su salida por el horizonte en los equinoccios.

2. Para el segundo objetivo podemos determinar que las estructuras 101 y 103 no están alineadas con respecto al solsticio de invierno ya que el acimut entre las estructuras 101 y 103 es de $138^{\circ}$ que no corresponde a ningún evento solar.

3. En el segundo objetivo también se buscaba determinar que las estructuras 101 y 104 estuvieran alineadas con el solsticio de verano lo cual tampoco podemos determinar su alineación ya que el acimut entre las estructuras 101 y 104 es de $37.61^{\circ}$ que no corresponde a ningún evento solar.

4. Envista de que el objetivo era encontrar alineamientos en el lugar, podemos concluir que si se encontraron alineamientos con otras estructuras como la 101 y 107 para el solsticio de verano, lastimosamente no podemos asegurar dicha alineación porque la estructura 107 fue destruida por completo, y en los registros bibliográficos no se encontró información referente a esta estructura, solamente en un plano con el cual se hizo los cálculos de medición aproximada de la altura de la montaña, en cuanto a las estructuras 101 y 111 también parece haber un alineamiento con el solsticio de invierno pero tampoco se puede asegurar por las mismas razones de la estructura 107.

5. En cuanto a las comparaciones con el programa del Stellarium del año 400 a. C y 2014 d. C., se observa una muy cercana similitud de acimut entre los diferentes eventos solares de equinoccios y solsticios.

\section{AGRADECIMIENTOS}

Un agradecimiento a especial al Profesor Cesar Rodríguez por su apoyo en la toma de datos, a Bertilio Amaya por su colaboración como guía de campo y a David Wiliams por su aporte de apoyo logístico. 


\section{BIBLIOGRAFÍA}

Mandeville, J. (1997). "Programa Arqueologico de Yarumela, temporada 1983: descubrimientos y analisis". YAXKIN, XV, edicion extraordinaria 1990-1994, 5-18.

Astronomy Education At the University of Nebraska- Lincolh. (2010). Astronomy Simulations and Animations, your player version: WIN 16,0,0,305. Recuperado de Flash animations and simulations for astronomy education. Topics include seasons, moon phases, coordinate systems, light, and more.: http://astro.unl.edu/classaction/animations/coordsmotion/sunmotions.html

Berrecoso, M., Ramírez , M. E., Enríquez Salamanca, J. M., \& Pérez Peña, A. (2003). Notas y apuntes de trigonometria esférica y astronomía de posición. Cádiz, España, Cádiz: Servicio de publicaciones de la Universidad de Cádiz.

Dixon, B. (1991). " La Arquitectura del periodo Formativo y la competencia del estado social en Yarumela, Honduras. YAXKIN, XV,edicion extraordinaria 1990-1994, 19-48.

Mandeville, J. (1986). "Proyecto Arqueologico Valle de Comayagua: Investigaciones en Yarumela-Chical. YAXKIN, IX N²,1986, 17-41.

Mandeville, J. L. (1997). "Programa Arqueologico de Yarumela, temporada 1983: descubrimientos y analisis". YAXKIN, XV, edicion extraordinaria 1990-1994, 5-18. 